\title{
Guided bone regeneration at zirconia and titanium dental implants: a pilot histological investigation
}

Benic, Goran I ; Thoma, Daniel S ; Sanz-Martin, Ignacio ; Munoz, Fernando ; Hämmerle, Christoph H F ; Cantalapiedra, Antonio ; Fischer, Jens ; Jung, Ronald E

\begin{abstract}
AIM: To test whether guided bone regeneration (GBR) of peri-implant defects at zirconia (ZrO2 ) implants differs from GBR at titanium (Ti) implants regarding the bone integration of the implant and of the grafting material. MATERIALS AND METHODS: Maxillary premolars and molars were extracted in seven dogs. After 5 months, four semi-saddle bone defects were created in each maxilla. Implant placement and simultaneous GBR were performed using the following randomly assigned modalities: (1) $\mathrm{ZrO} 2$ implant + deproteinized bovine bone mineral (DBBM) granules + a collagen membrane (CM), (2) ZrO2 implant + DBBM with 10\% collagen matrix + CM, (3) ZrO2 implant + DBBM block + CM, and (4) Ti implant + DBBM granules + CM. After 3 months, one central histological section of each site was prepared. Histomorphometrical assessments were performed evaluating the augmented area (AA) within the former bone defect (primary outcome), the area of new bone (NB), bone substitute (BS), and non-mineralized tissue (NMT) within AA in $\mathrm{mm} 2$. In addition, the distance between the most coronal bone-to-implant contact and the margin of the former bone defect (fBIC-DEF), and the bone-to-implant contact fraction (BIC) were measured in mm. RESULTS: AA measured $8.6 \pm 4.0 \mathrm{~mm} 2$ for $\mathrm{ZrO} 2$ implant + DBBM granules, $4.7 \pm 1.6 \mathrm{~mm} 2$ for $\mathrm{ZrO} 2 \mathrm{implant}+\mathrm{DBBM}$-collagen, $5.1 \pm 1.9 \mathrm{~mm} 2$ for ZrO2 implant + DBBM block, and $7.6 \pm 2.8 \mathrm{~mm} 2$ for Ti implant + DBBM granules. There were no statistically significant differences between the treatment modalities $(\mathrm{P}>0.05)$. NB reached $2.0 \pm 1.7 \mathrm{~mm} 2$ for $\mathrm{ZrO} 2$ implant + DBBM granules, $0.9 \pm 0.9 \mathrm{~mm} 2$ for $\mathrm{ZrO} 2$ implant + DBBM-collagen, $2.1 \pm 0.9 \mathrm{~mm} 2$ for $\mathrm{ZrO} 2$ implant + DBBM block, and $0.8 \pm 0.6 \mathrm{~mm} 2$ for $\mathrm{Ti}$ implant + DBBM granules. fBIC-DEF amounted to $2.1 \pm 1.7 \mathrm{~mm} 2$ for $\mathrm{ZrO} 2$ implant + DBBM granules, to $2.7 \pm$ $1.1 \mathrm{~mm} 2$ for ZrO2 implant + DBBM-collagen, to $2.9 \pm 1.2 \mathrm{~mm} 2$ for ZrO2 implant + DBBM block, and to $3.4 \pm 0.4$ $\mathrm{mm} 2$ for Ti implant + DBBM granules. BIC measured $70 \pm 19 \%$ for ZrO2 implant + DBBM granules, $69 \pm 22 \%$ for ZrO2 implant + DBBM-collagen, $77 \pm 30 \%$ for ZrO2 implant + DBBM block, and $66 \pm 27 \%$ for Ti implant + DBBM granules. CONCLUSIONS: The findings of the present pilot study suggest that zirconia and titanium implants grafted with DBBM granules and covered with a collagen membrane do not perform differently regarding the augmented ridge contour, the NB formation, and the implant osseointegration.
\end{abstract}

DOI: https://doi.org/10.1111/clr.13030

Posted at the Zurich Open Repository and Archive, University of Zurich

ZORA URL: https://doi.org/10.5167/uzh-139620

Journal Article

Accepted Version

Originally published at:

Benic, Goran I; Thoma, Daniel S; Sanz-Martin, Ignacio; Munoz, Fernando; Hämmerle, Christoph H F; Cantalapiedra, Antonio; Fischer, Jens; Jung, Ronald E (2017). Guided bone regeneration at zirconia and titanium dental implants: a pilot histological investigation. Clinical Oral Implants Research, 28(12):1592-1599. 
DOI: https://doi.org/10.1111/clr.13030 


\section{Guided bone regeneration at zirconia and titanium dental implants: a pilot histologic investigation}

\section{Goran I. Benic ${ }^{1}$, Daniel S. Thoma ${ }^{1}$, Ignacio M. Sanz ${ }^{2}$, Fernando Munoz ${ }^{3}$, Christoph H.F. Hämmerle ${ }^{1}$, Antonio Cantalapiedra ${ }^{3}$, Jens Fischer ${ }^{4}$, Ronald E. Jung ${ }^{1}$,}

1: Clinic of Fixed and Removable Prosthodontics and Dental Material Science, Center of Dental Medicine, University of Zurich, Switzerland

2: Faculty of Odontology, Universidad Complutense of Madrid, Madrid, Spain

3: Faculty of Veterinary, University of Santiago de Compostela, Spain

4: Division of Dental Materials and Engineering, University of Basel, Basel, Switzerland

Key words: alveolar ridge augmentation, alveolar ridge defect, animal study, bone, dental implants, zirconium dioxide, zirconia, membrane, bone substitute, bone graft, guided bone regeneration, histology

Running title: $\mathrm{GBR}$ at $\mathrm{ZrO}_{2}$ vs. Ti implants

Corresponding author:

PD Dr. Goran I. Benic

Clinic of Fixed and Removable Prosthodontics and Dental Material Science

Center of Dental Medicine

University of Zurich

Plattenstrasse 11

8032 Zurich, Switzerland

Tel: +416343252

E-mail: goran.benic@zzm.uzh.ch 


\section{Abstract}

Aim: To test whether guided bone regeneration (GBR) of peri-implant defects at zirconia $\left(\mathrm{ZrO}_{2}\right)$ implants differs from GBR at titanium $(\mathrm{Ti})$ implants regarding the bone integration of the implant and of the grafting material.

Material and methods: Maxillary premolars and molars were extracted in 7 dogs. After 5 months, four semi-saddle bone defects were created in each maxilla. Implant placement and simultaneous GBR was performed using the following randomly assigned modalities: (1) $\mathrm{ZrO}_{2}$ implant + deproteinized bovine bone mineral (DBBM) granules + a collagen membrane (CM), (2) $\mathrm{ZrO}_{2}$ implant + DBBM with $10 \%$ collagen matrix $+\mathrm{CM}$, (3) $\mathrm{ZrO}_{2}$ implant + DBBM block + CM, and (4) Ti implant + DBBM granules + CM. After 3 months, one central histological section of each site was prepared. Histomorphometrical assessments were performed evaluating the augmented area (AA) within the former bone defect (primary outcome), the area of new bone (NB), bone substitute (BS), and non-mineralized tissue (NMT) within AA in $\mathrm{mm}^{2}$. In addition, the distance between the most coronal bone-to-implantcontact and the margin of the former bone defect (fBIC-DEF), and the bone-to-implant contact fraction (BIC) were measured in $\mathrm{mm}$.

Results: AA measured $8.6 \pm 4.0 \mathrm{~mm}^{2}$ for $\mathrm{ZrO}_{2}$ implant+DBBM granules, $4.7 \pm 1.6 \mathrm{~mm}^{2}$ for $\mathrm{ZrO}_{2}$ implant+DBBM/collagen, $5.1 \pm 1.9 \mathrm{~mm}^{2}$ for $\mathrm{ZrO}_{2}$ implant+DBBM block, and 7.6 \pm 2.8 $\mathrm{mm}^{2}$ for Ti implant+DBBM granules. There were no statistically significant differences between the treatment modalities $(P>0.05)$. NB reached $2.0 \pm 1.7 \mathrm{~mm}^{2}$ for $\mathrm{ZrO}_{2}$ implant+DBBM granules, $0.9 \pm 0.9 \mathrm{~mm}^{2}$ for $\mathrm{ZrO}_{2}$ implant+DBBM/collagen, $2.1 \pm 0.9 \mathrm{~mm}^{2}$ for $\mathrm{ZrO}_{2}$ implant+DBBM block, and $0.8 \pm 0.6 \mathrm{~mm}^{2}$ for Ti implant+DBBM granules. fBIC-DEF amounted to $2.1 \pm 1.7 \mathrm{~mm}^{2}$ for $\mathrm{ZrO}_{2}$ implant+DBBM granules, to $2.7 \pm 1.1 \mathrm{~mm}^{2}$ for $\mathrm{ZrO}_{2}$ implant+DBBM/collagen, to $2.9 \pm 1.2 \mathrm{~mm}^{2}$ for $\mathrm{ZrO}_{2}$ implant+DBBM block, and to $3.4 \pm 0.4 \mathrm{~mm}^{2}$ for Ti implant+DBBM granules. BIC measured $70 \pm 19 \%$ for $\mathrm{ZrO}_{2}$ implant+DBBM granules, $69 \pm 22 \%$ for $\mathrm{ZrO}_{2}$ implant+DBBM/collagen, $77 \pm 30 \%$ for $\mathrm{ZrO}_{2}$ implant+DBBM block, and $66 \pm 27 \%$ for Ti implant+DBBM granules.

Conclusions: The findings of the present pilot study suggest that zirconia and titanium implants grafted with DBBM granules and covered with a collagen membrane do not perform differently regarding the augmented ridge contour, the new bone formation and the implant osseointegration. 


\section{Introduction}

Ceramic dental implants made of zirconium dioxide (zirconia) were brought to the market as an alternative to metal implants made of titanium and titanium-alloys. The clinical use of zirconia implants is currently increasing due to the general trend for metal-free solutions and tooth-colored reconstruction materials.

Previous investigations demonstrated the high biocompatibility and the promising mechanical properties of zirconia, such as high fracture toughness, high flexural strength and hardness, resistance to corrosion, and low thermal conductivity (Hisbergues et al. 2009) (Ichikawa et al. 1992; Kohal et al. 2009; Piconi et al. 1998). Several animal studies did not find differences in the degree of osseointegration between zirconia and titanium implants under different loading conditions (Gahlert et al. 2012; Gahlert et al. 2009; Koch et al. 2010; Kohal et al. 2004; Schliephake et al. 2010; Stadlinger et al. 2010; Thoma et al. 2015). A recent systematic review analyzed the preclinical investigations of the bone-to-implant contact (BIC) values of zirconia and titanium implants (Manzano et al. 2014). The review included 19 studies and concluded that in most of the trials BIC values did not significantly differ between zirconia and titanium implants.

Despite the existence of several commercially available zirconia implant systems, not many clinical studies documenting the use of zirconia implants are available yet. Recent clinical trials investigating zirconia implants found successful tissue integration and high survival rates after short-term observation periods (Cannizzaro et al. 2010; Jung et al. 2016; Kohal et al. 2012; Kohal et al. 2013; Oliva et al. 2010; Payer et al. 2013; Payer et al. 2015). In one prospective study, the investigated zirconia implant fulfilled the success criteria that have been proposed for titanium implants (Albrektsson \& Isidor 1993; Jung et al. 2016).

Guided bone regeneration (GBR) procedure is routinely performed in daily practice to regenerate missing hard tissue volume prior to or simultaneously with implant placement (Benic \& Hammerle 2014). There is a large body of clinical evidence documenting that survival rates of titanium implants placed in conjunction with GBR are similar to those of titanium implants placed into native bone (Benic et al. 2009; Jung et al. 2013; Zitzmann et al. 2001; Zumstein et al. 2012). Currently, the application of xenografts in combination with resorbable collagen membranes is the most widely used and well-documented method for the augmentation of peri-implant defects (Chiapasco \& Zaniboni 2009; Jensen \& Terheyden 2009). GBR with particulate xenograft and collagen membrane renders high percentage of apico-coronal defect fill and long-term stability of the augmented hard tissue (Buser et al. 2013; Jung et al. 2015). Moreover, it was demonstrated that GBR of peri-implant bone defects renders a long-term stable increase in the peri-implant mucosal contour (Benic et al. 
2016a). However, to date, there is very little preclinical and clinical evidence documenting the use of GBR concomitant with the placement of zirconia implants.

Therefore, the primary aim of the present pilot study was to test whether or not GBR of peri-implant defects at zirconia implants by means of xenogenic bone substitutes and collagen membranes differs from GBR at titanium implants with regard to hard tissue integration of the implant and of the grafting material. Moreover, at zirconia implants different particulate and non-particulate bone substitutes were compared for GBR in combination with collagen membranes. 


\section{Materials and methods}

This article was written in accordance with the ARRIVE (Animal research: Reporting of in vivo experiments) guidelines (Kilkenny et al. 2010).

\section{Animals}

Seven male adult beagle dogs (age $20 \pm 3$ months, mean weight $16.8 \mathrm{~kg}$ ) (Isoquimen, Barcelona, Spain) were included in this trial. All animals presented a fully erupted permanent dentition.

The animals were subjected to surgeries and housed in the Animal Experimentation Service Facility at the Veterinary Hospital Rof Codina, Lugo, Spain. Prior to the start of the study, the experimental protocol was approved by the Ethical Committee Rof Codina Foundation. All the procedures were performed according to Spanish and European Union regulations about care and use of research animals. The dogs were monitored daily for the duration of the study by a veterinarian accredited in laboratory animal science. The animals were housed in a group kennel with indoor and outdoor areas. The indoor area presented a controlled temperature of $20-22^{\circ} \mathrm{C}$ with natural light and air renewal. During the study period, the animals received a soft-food diet and water ad libitum. The trial started after a 3-week adaption period for the animals.

\section{Study design and randomization}

This investigation was designed as a randomized controlled trial with intra-subject control for the comparison of four treatment modalities. The study was performed in two surgical phases including (1) tooth extraction and (2) implant placement with simultaneous GBR of acute-type peri-implant bone defects (four defects per animal).

The experimental sites were randomly allocated to one of the four treatment procedures according to a computer-generated randomization list. Allocation to the treatment was concealed by means of sealed envelopes until the time of the GBR procedure.

\section{Surgical procedures}

The investigators participating in the study were experienced in implant placement and guided bone regeneration procedures. The surgical part of this study was conducted from October 2011 to May 2012.

All surgical procedures were performed under general anesthesia of the animals. The dogs were pre-medicated with medetomidine (20 $\mu \mathrm{g} / \mathrm{kg} / \mathrm{i} . \mathrm{m}$.; Esteve, Barcelona, Spain) and morphine (0.4 mg/kg/i.m., Morfina Braun 2\%; B. Braun Medical, Barcelona, Spain). The 
anesthesia was initiated by propofol ( $2 \mathrm{mg} / \mathrm{kg} / \mathrm{i} . \mathrm{v}$.; Propovet, Abbott Laboratories, Kent, UK), and maintained by inhalation of an $\mathrm{O}_{2}$ and $2.5-4 \%$ isoflurane mixture (Isobavet, ScheringPlough, Madrid, Spain). A local anesthesia composed of lidocaine and adrenaline (Anesvet ${ }^{\circledR}$, Ovejero, Leon, Spain) was used to reduce peri-operative pain and bleeding. During anesthesia, the dogs were monitored by a veterinarian, applying electrocardiography, capnography, pulsioxymetry, and blood pressure measurements. After the surgery, atipamezol ( $50 \mu \mathrm{g} / \mathrm{kg} / \mathrm{i} . \mathrm{m}$.; Esteve) was administered to revert the effects of the medetomidine.

Postoperatively, pain was controlled with morphine ( $0.3 \mathrm{mg} / \mathrm{kg} / \mathrm{i} . \mathrm{m}$.) for $24 \mathrm{~h}$ and, subsequently, with meloxicam $(0.1 \mathrm{mg} / \mathrm{kg} /$ s.i.d/p.o.; Metacam, Boehringer Ingelheim, Barcelona, Spain) for 3 days. Antibiotics (amoxicillin 22 mg/kg/s.i.d./s.c.; Amoxoil retard, Syva, Leon, Spain) were administrated for 7 days.

During the first two postoperative weeks, the oral mucosa and the teeth were disinfected three times a week by using gauzes soaked in a $0.12 \%$ chlorhexidine solution (Perio-Aid Tratamiento ${ }^{\circledR}$, Dentaid, Barcelona, Spain). Subsequently, a toothbrush and a $0.2 \%$ chlorhexidine gel (Chlorhexidine Bioadhesive Gel, Lacer, Barcelona, Spain) were used for plaque control.

\section{Surgery 1 (tooth extraction)}

The maxillary second, third and fourth premolars (P2, P3, P4) and first molars (M1) were bilaterally sectioned by using fissure burs and extracted with dental elevators and forceps. Mucosal margins were adapted by means of resorbable sutures (Vicryl ${ }^{\circledR} 5-0$, Ethicon, Issy Les Moulineaux Cedex, France).

Surgery 2 (defect preparation, implant placement, and GBR)

Preparation of defects, implant placement and GBR of peri-implant bone defects were conducted 5 months after tooth extraction. Following a mid-crestal incision from the region of $\mathrm{P} 2$ to $\mathrm{M} 2$ and a vertical releasing incision in the region of $\mathrm{P} 2$, the mucoperiosteal flaps were elevated. The coronal portion of the edentulous ridge was flattened in the region of P4 and M1.

On each side of the maxilla, two implant beds with a diameter of $4 \mathrm{~mm}$ were prepared $3 \mathrm{~mm}$ from each other and $2 \mathrm{~mm}$ lingual to the buccal wall of the alveolar ridge.

Subsequently, a standardized semi-saddle-type bone defect was prepared at each implant bed by removing the buccal wall of the alveoloar ridge. The bone defects measured $4 \mathrm{~mm}$ mesio-distally, $4 \mathrm{~mm}$ apico-coronally, and $2 \mathrm{~mm}$ bucco-orally (Fig. 1). All the osteotomy procedures were performed under irrigation with sterile $0.9 \%$ saline. 
Three test zirconia bone level implants and one control titanium bone level implant were placed in each maxilla obtaining primary stability. A prototype bone level implant made of yttrium-stabilized zirconia was used in this study (vitaclinical, VITA Zahnfabrik, Bad Säckingen, Germany). The surface morphology of the zirconia implant was created by sandblasting, acid etching with hydrofluoric acid, and subsequent annealing. The obtained surface roughness was in the range of $\mathrm{Ra}=1.2 \mu \mathrm{m}$ ( Fischer et al. 2016). Titanium bone level implant was used as control (OsseoSpeed ${ }^{\text {TM }}$ S, ASTRA TECH Implant System, DENTSPLY Implants, Mannheim, Germany). All the implants exhibited a diameter of $4 \mathrm{~mm}$ and a length of $8 \mathrm{~mm}$. The apico-coronal position of the implant shoulder coincided with the most coronal level of the palatal bone wall (Fig. 1). Titanium closure screws (DENTSPLY Implants) were placed on the titanium implants.

The defects were randomly allocated to receive one of the following treatments (Fig. 2):

- $\mathrm{ZrO}_{2}$ implant+DBBM granules: zirconia implant (vitaclinical) + particulated deproteinized bovine bone material (DBBM) (Bio-Oss ${ }^{\circledR}$ granules $0.25-1 \mathrm{~mm}$, Geistlich Pharma AG, Wolhusen, Switzerland) + a non-cross-linked porcine collagen membrane (CM) (Bio-Gide ${ }^{\circledR}$, Geistlich Pharma AG)

- $\mathrm{ZrO}_{2}$ implant+DBBM-collagen: zirconia implant (vitaclinical) + DBBM with collagen matrix (Bio-Oss ${ }^{\circledR}$ collagen, Geistlich Pharma AG) + CM

- $\mathrm{ZrO}_{2}$ implant+DBBM block: zirconia implant (vitaclinical) + DBBM cancellous block: (Bio-Oss ${ }^{\circledR}$ spongiosa block, Geistlich Pharma AG) + CM

- $\quad$ Ti implant+DBBM granules: titanium implant (DENTSPLY Implants) + particulated $\mathrm{DBBM}+\mathrm{CM}$

The bone substitutes were applied to achieve $1 \mathrm{~mm}$ of over-contour with respect to the buccal surface of the alveolar ridge. The DBBM blocks and DBBM-collagen were individually shaped and adapted to fit the bone defects. Prior to the application, all bone substitutes were hydrated with sterile $0.9 \%$ saline. CM was applied to cover the bone substitute and overlap the walls of the defect by at least $2 \mathrm{~mm}$ (Fig. 2). No screws or pins for stabilization of blocks and membranes were used.

The periosteum of the buccal flap was relieved along its base, and primary wound closure was accomplished by placing horizontal mattress and interrupted sutures made of ePTFE (Gore-Tex ${ }^{\circledR}$ sutures 5-0; W.L. Gore \& Associates Inc., Flagstaff, AZ, USA). The sutures were removed after 2 weeks. 


\section{Retrieval of specimens}

Three months after implant placement and GBR, the animals were sedated with medetomidine (30 $\mu \mathrm{g} / \mathrm{kg} / \mathrm{i} . \mathrm{m}$.; Esteve) and subsequently killed with an overdose of sodium pentobarbital (40-60 mg/kg/i.v., Dolethal, Vetoquinol, France). The maxillae were dissected, and the experimental specimens were retrieved with intact soft tissues.

\section{Histological preparation}

Fixation of the specimens was performed in buffered $4 \%$ formaldehyde solution. The specimens were dehydrated using ethanol solutions of increasing concentrations and subsequently embedded in a methyl methacrylate resin (Sigma-Aldrich M55909-1L, SigmaAldrich, Buchs, Switzerland). Radiographs were taken of each site to accurately determine the section planes. One bucco-oral section through the central axis of the implant was prepared from each site. The tissue blocks were cut into $200-\mu m$-thick sections using a diamond band saw (Exakt Apparatebau, Norderstedt, Germany). The sections were ground and polished to a thickness of 60-80 $\mu$ m (Exakt Apparatebau) (Donath \& Breuner 1982). The sections were stained with toluidine blue (Schenk et al. 1984) (Fig. 3).

\section{Histomorphometric analysis}

Histomorphometrical analysis was performed by one investigator that was unaware of the specific experimental conditions. A light microscope (Microscope Leica DM6000, Leica Mikrosysteme, Wetzlar, Germany) connected with a digital color camera (Leica DFC 450 (Leica Mikrosysteme) was used for image capturing. For histomorphometrical analysis, digital images were evaluated using an image analysis software (Leica Application Suite V4.3, Leica Mikrosysteme).

The following reference points were identified on the buccal surface of each implant: the implant shoulder (IS), the most coronal level of bone in contact with the implant (fBIC) and the apical margin of the former bone defect (DEF).

For each section, the following variables were assessed:

- the augmented area $(\mathrm{AA})\left(\mathrm{mm}^{2}\right)$ within the former bone defect at the buccal aspect (primary outcome) (Fig. 4)

- the area of new mineralized bone (NB) $\left(\mathrm{mm}^{2}\right)$, residual bone substitute (BS) $\left(\mathrm{mm}^{2}\right)$, and non-mineralized tissue (NMT) $\left(\mathrm{mm}^{2}\right)$ within AA (Fig. 4) 
- the apico-coronal distance between the most coronal level of bone in contact with the implant and the apical margin of the former bone defect (fBIC-DEF) (Fig. 5)

- bone-to-implant contact fraction (BIC) $(\%)$ in the $2 \mathrm{~mm}$-long region-of-interest apical to the margin of the former bone defect.

\section{Statistical analysis}

The animal was chosen as the unit for the statistical analysis. The data were reported by using means, standard deviations (SD), ranges, 95\% confidence intervals (CI), medians, and interquartile ranges (IQR) (SPSS software; SPSS Inc., Chicago, IL, USA). The Wilcoxon signed rank test was applied to detect differences between the treatments ( $R$ software; $R$ Foundation, Vienna, Austria). The results of tests with P-values $\leq 0.05$ were considered statistically significant. Due to the exploratory nature of this study, no sample size calculation and no correction for multiple testing were performed. 


\section{Results}

All animals remained healthy during the study period and no adverse events were observed. One histological specimen from the $\mathrm{ZrO}_{2}$ implant+DBBM-collagen and one specimen from the $\mathrm{ZrO}_{2}$ implant+DBBM block groups were excluded from the analysis due to the erroneous placement of the histologic section. One $\mathrm{ZrO}_{2}$ implant augmented with DBBM/collagen did not integrate and it was excluded from the analysis. A total of $7 \mathrm{ZrO}_{2}$ implant+DBBM granules, $5 \mathrm{ZrO}_{2}$ implant+DBBM/collagen, $6 \mathrm{ZrO}_{2}$ implant+DBBM block, and $7 \mathrm{Ti}$ implant+DBBM granules sites were available for the histomorphometrical analysis.

Augmented area (AA) amounted to $8.6 \pm 4.0 \mathrm{~mm}^{2}$ (SD) for $\mathrm{ZrO}_{2}$ implant+DBBM granules, $4.7 \pm 1.6 \mathrm{~mm}^{2}$ (SD) for $\mathrm{ZrO}_{2}$ implant+DBBM-collagen, $5.1 \pm 1.9 \mathrm{~mm}^{2}$ (SD) for $\mathrm{ZrO}_{2}$ implant+DBBM block, and $7.6 \pm 2.8 \mathrm{~mm}^{2}$ (SD) for Ti implant+DBBM granules. The sites augmented with DBBM granules resulted in higher mean values in comparison to the sites treated with DDBM/collagen and DBBM block. There were, however, no statistically significant differences between the treatment modalities $(P>0.05)$ (Table 1, Fig. 6).

New mineralized bone (NB) measured $2.0 \pm 1.7 \mathrm{~mm}^{2}$ (SD) for $\mathrm{ZrO}_{2}$ implant+DBBM granules, $0.9 \pm 0.9 \mathrm{~mm}^{2}$ (SD) for $\mathrm{ZrO}_{2}$ implant+DBBM-collagen, $2.1 \pm 0.9 \mathrm{~mm}^{2}$ (SD) for $\mathrm{ZrO}_{2}$ implant+DBBM block, and $0.8 \pm 0.6 \mathrm{~mm}^{2}(\mathrm{SD})$ for Ti implant+DBBM granules (Table 1, Fig. $6)$.

Residual bone substitute (BS) reached $2.3 \pm 1.6 \mathrm{~mm}^{2}$ (SD) for $\mathrm{ZrO}_{2}$ implant+DBBM granules, $1.9 \pm 1.7 \mathrm{~mm}^{2}$ (SD) for $\mathrm{ZrO}_{2}$ implant+DBBM-collagen, $1.0 \pm 0.6 \mathrm{~mm}^{2}$ (SD) for $\mathrm{ZrO}_{2}$ implant+DBBM block, and $2.4 \pm 1.6 \mathrm{~mm}^{2}$ (SD) for Ti implant+DBBM granules. The sites augmented with DBBM granules reached higher mean values than the sites treated with DBBM/collagen and DBBM block (Table 1, Fig. 6).

With regard to the distance between the most coronal level of bone in contact with the implant and the apical margin of the former bone defect (fBIC-DEF), the values amounted to $2.1 \pm 1.7 \mathrm{~mm}^{2}$ (SD) for $\mathrm{ZrO}_{2}$ implant+DBBM granules, to $2.7 \pm 1.1 \mathrm{~mm}^{2}$ (SD) for $\mathrm{ZrO}_{2}$ implant+DBBM-collagen, to $2.9 \pm 1.2 \mathrm{~mm}^{2}$ (SD) for $\mathrm{ZrO}_{2}$ implant+DBBM block, and to $3.4 \pm 0.4 \mathrm{~mm}^{2}$ (SD) for Ti implant+DBBM granules (Table 1).

Bone-to-implant contact measured $70 \pm 19 \%$ (SD) for $\mathrm{ZrO}_{2}$ implant+DBBM granules, $69 \pm 22 \%$ (SD) for $\mathrm{ZrO}_{2}$ implant+DBBM-collagen, $77 \pm 30 \%$ (SD) for $\mathrm{ZrO}_{2}$ implant+DBBM block, and $66 \pm 27 \%$ (SD) for Ti implant+DBBM granules (Table 1). 


\section{Discussion}

The results of the present study indicated that the application of DBBM granules and CM for GBR of peri-implant defects at zirconia implant did not differ from GBR at titanium implants with regard to the implant osseointegration, the dimension of the augmented ridge, and the new bone formation. For peri-implant defects at zirconia implants, the application of DBBM granules and CM resulted in higher mean width of the ridge in comparison to the use of DBBM-collagen and DBBM blocks in combination with $\mathrm{CM}$. Due to the exploratory nature of the present trial with small sample size, these findings need to be interpreted with caution.

The findings regarding the osseointegration of zirconia and titanium implants from the present study are in accordance with previous investigations. Several animal studies did not find differences in the amount of osseointegration between zirconia and titanium implants under different loading conditions (Gahlert et al. 2012; Gahlert et al. 2009; Koch et al. 2010; Kohal et al. 2004; Schliephake et al. 2010; Stadlinger et al. 2010; Thoma et al. 2015). A recent systematic review included 19 preclinical histologic investigations of the bone-toimplant contact at zirconia and titanium implants (Manzano et al. 2014). It was concluded that in most of the trials bone-to-implant contact values did not differ between zirconia and titanium implants.

In the present study, the apico-coronal distance between the most coronal bone-toimplant contact and the apical wall of the former bone defect was measured. The assessment of bone growth along the originally exposed implant surface can be interpreted as a measure of vertical osteoconductivity of the implant surface. The bone growth along the exposed buccal surface of the titanium implant reached in average more coronal levels in comparison to the zirconia implants. To our knowledge, this is the first trial comparing the osteogenic surface properties of the exposed zirconium and titanium implants.

With respect to the dimension of the augmented ridge, GBR with DBBM granules and $\mathrm{CM}$ at zirconia implants did not differ from GBR at titanium implants. In contrast, the mean values of the ridge contour at zirconia implants differed when comparing particulate and non-particulate grafting materials. Particulate DBBM in combination with CM reached the highest mean value in ridge dimensions, followed by DBBM block and DBBM-collagen in combination with $\mathrm{CM}$. The higher mean dimension of the ridge augmented with particulate DBBM was due to the higher amount of residual bone substitutes and non-mineralized tissue. Based on this finding, it can be deduced that the contour of the augmented ridge was mainly influenced by the properties of the grafting materials used for GBR.

In all the experimental sites, no complete augmentation of the initial bone defect could be achieved. This result could be partially explained by the displacement of the 
mechanically unstable grafting materials and collagen membranes during the healing period. It is known that particulated bone substitutes in combination with $\mathrm{CM}$ are sub-optimal for the augmentation of non-contained bone defects due to the unfavorable mechanical properties

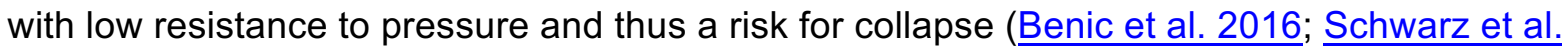
2007; Strietzel et al. 2006; Zellin et al. 1995). In this context, it is surprising that sites augmented with DBBM blocks and DBBM-collagen resulted in lower mean values in ridge contour compared to the sites augmented with particulated DBBM.

The low value of the augmented area achieved with DBBM block in combination with $\mathrm{CM}$ can be partially explained by the intra- and post-operative fractures of DBBM blocks and subsequent displacement of the block fragments. Intra-operatively, DBBM block was prone to fractures, thereby not permitting "press-fit" adaptation and mechanical anchorage within the box-shaped defects. In a recent in-vitro study, the volume stability of particulated and block bone substitutes in combination with CM for GBR of peri-implant defects was investigated by means of cone-beam computed tomography (СBCT) (Mir-Mari et al. 2016b). It was found that wound closure and flap suturing always induced a considerable displacement of the particulated grafting material resulting in a partial collapse of the CM. In contrast, the sites augmented with block bone substitutes exhibited less collapse of the membranes. The investigators concluded that the ability to maintain the contour of the augmented region during wound closure and flap suturing was significantly enhanced when using a block bone substitute in comparison to GBR with particulated material. These findings were confirmed by an animal study that was designed to assess the performance of xenogenic blocks and granules (Benic et al. 2016). In this study, equine-derived blocks, DBBM blocks and particulated DBBM were used in combination with CM for GBR of large peri-implant defects. The equine-derived bone block substitute in combination with $\mathrm{CM}$ reached the highest mean values in ridge dimensions, followed by DBBM block and DBBM granulate in combination with CM. A comparison of particulated and block DBBM for horizontal augmentation of chronic-type bone defects was performed in another preclinical study (Schwarz et al. 2008). In this trial, the bone substitute and CM were used alone or with an addition of growth factors. After 3 and 8 weeks, samples were prepared for histological analysis. When assessing the width of the augmented ridge, block of DBBM stabilized by titanium screws appeared to perform better in comparison with particulated DBBM. The results of these studies are not in accordance with the findings regarding GBR with DBBM block from the present trial. The differences in the results between the trials may be explained by the different localization and size of the experimental defects.

In the present study the lowest mean value of the augmented ridge was achieved by DBBM-collagen. This composite material consists of $90 \%$ DBBM particles embedded in a $10 \%$ resorbable collagen matrix of porcine origin. The addition of collagen aims to facilitate 
the clinical handling and to achieve a stabilization of the graft (Wong \& Rabie 2010). The influence of the mechanical stability of DBBM-collagen on the performance of GBR of periimplant defects was investigated in a recent in-vitro study (Mir-Mari et al. 2016a). This study tested whether GBR with DBBM-collagen in addition to the DBBM granules differs from GBR with DBBM granules with regard to the volume stability of the augmented region during flap closure. The investigators concluded that the addition of DBBM-collagen soft-type block to DBBM granules, covered by CM and fixed with pins, significantly improved the horizontal volume stability of the augmented region during wound closure. The findings of this in-vitro study are in contradiction with results from the present trial. The poor result in the maintenance of the augmented ridge with DBBM-collagen from the present study could be explained by the biodegradation of the collagen matrix and a subsequent displacement of the DBBM particles. It is striking that, even though DBBM-collagen has been widely used for different clinical indications, there is insufficient evidence regarding the use of DBBMcollagen for GBR of peri-implant defects. To our knowledge, there are no previous studies comparing the use of DBBM with and DBBM without collagen matrix for GBR of peri-implant defects regarding the volume stability of the augmented area. A recent randomized controlled trial compared the dimensional changes after ridge preservation with particulated DBBM and DBBM-collagen (Nart et al. 2016). CBCT were taken at tooth extraction and at 5 months. Both treatments resulted in a significant ridge reduction in width and height. When comparing the treatment groups, although there were no statistically significant differences, DBBM group showed less dimensional changes compared to the DBBM-collagen group. While in the DBBM group ridge width decreased $0.91 \mathrm{~mm}$, a mean reduction of $1.53 \mathrm{~mm}$ was measured in the DBBM-collagen group. These findings are in accordance with the results of the augmented ridge dimensions from the present study.

Due to the small sample size and considerable variations of the data, the results of the present study have to be interpreted with caution. Another important limitation of the present trial is the lack of the negative control group. Consequently, it was not possible to assess the degree of the spontaneous bone healing at the experimental maxillary acute-type peri-implant defects. The model of an acute-type defect used in this study represents another methodological shortcoming, as this type of defect is not considered to adequately simulate the clinical reality. Nevertheless, this study provides valuable data on the bone integration of zirconia implants grafted with particulate and non-particulate bone substitutes for GBR. Further controlled clinical trials are needed to examine the clinical implications of the findings from the present preclinical study. 


\section{Conclusions}

The findings of the present study suggest that for guided bone regeneration of periimplant bone defects:

- Zirconia and titanium implants grafted with DBBM granules and covered with collagen membranes did not perform differently in terms of dimension of the augmented ridge, new bone formation and implant osseointegration.

- For peri-implant defects at zirconia implants, the application of DBBM granules and collagen membranes revealed the most favorable results regarding the augmented ridge contour. 


\section{Acknowledgments and conflict of interest}

The investigators gratefully acknowledge the team at the Veterinary Hospital Rof Codina, Lugo, Spain for animal care, Ms. Sonja Hitz (Clinic of Fixed and Removable Prosthodontics and Dental Material Science, Center of Dental Medicine, University of Zurich, Switzerland) for the histological preparation and analysis, Ms. Gisela Müller (Clinic of Fixed and Removable Prosthodontics and Dental Material Science, Center of Dental Medicine, University of Zurich, Switzerland) for assistance in preparing the manuscript, and Mr. Daniel Wiedemeier (Center of Dental Medicine, University of Zurich, Switzerland) for assistance in analyzing the data. This study was supported by the Clinic of Fixed and Removable Prosthodontics and Dental Material Science, Center of Dental Medicine, University of Zurich, Switzerland, and by a research grant from vitaclinical, a business unit of VITA Zahnfabrik, Bad Säckingen, Germany. Besides his appointment at the University of Basel, Prof. Fischer is an employee of VITA Zahnfabrik. The other authors do not report to have any conflict of interest to any products related to this study. 


\section{Figure legends}

Fig. 1. (a) Buccal and (b) occlusal views of the bone defects prior to guided bone regeneration.

Fig. 2. (a, b, c) GBR procedures with particulate deproteinized bovine bone mineral (DBBM) (left) and DBBM with collagen matrix (right) in combination with collagen membrane at zirconia implants. (d, e, f) GBR procedures with particulate DBBM in combination with collagen membrane at titanium implant (control) (left) and block DBBM in combination with collagen membrane at zirconia implant.

Fig. 3. Histological views (original magnification $x 12.5$ ): (a) zirconia implant with particulated deproteinized bovine bone material (DBBM) and collagen membrane (CM), (b) zirconia implant with DBBM-collagen and CM, (c) zirconia implant with DBBM block and CM, and (d) titanium implant with particulated DBBM and CM.

Fig 4. Histomorphometrical analysis of the augmented area (AA) within the former bone defect, the area of new mineralized bone (blue surface) within $A A$, and the area of residual bone substitute (red surface) within AA.

Fig 5. (a) Histomorphometrical measurement of the distance between the most coronal level of bone in contact with the implant (fBIC) and the margin of the former bone defect (DEF) (original magnification $\times 25$ ). The red rectangle represents the extension of the following high-magnification image (b) The most coronal level of bone in contact with the buccal implant surface marked by the blue arrow (original magnification $\times 500$ ).

Fig 6. Plots representing the mean values of the surfaces (in $\mathrm{mm}^{2}$ ) of new bone (NB), residual bone substitute (BS), and non-mineralized tissue (NMT) within the augmented area (AA) for different treatment modalities. The total sum of NB, BS and NMT corresponds to AA. 


\section{Table legends}

Table. 1. Results of the (a) histomorphometrical analysis; (b) statistical tests for comparisons between the treatment modalities. 


\section{References}

Albrektsson, T. \& Isidor, F. (1993) Consensus report of session iv Lang, N.P. \& Karring, T., eds. Proceedings of the 1st european workshop on periodontology, London: Quintessence.: 365-369.

Benic, G. I. \& Hammerle, C. H. (2014) Horizontal bone augmentation by means of guided bone regeneration. Periodontology 2000 66: 13-40.

Benic, G. I., Jung, R. E., Siegenthaler, D. W. \& Hammerle, C. H. (2009) Clinical and radiographic comparison of implants in regenerated or native bone: 5-year results. Clinical Oral Implants Research 20: 507-513.

Benic, G. I., Thoma, D. S., Munoz, F., Sanz Martin, I., Jung, R. E. \& Hammerle, C. H. (2016) Guided bone regeneration of peri-implant defects with particulated and block xenogenic bone substitutes. Clinical Oral Implants Research 27: 567-576.

Buser, D., Chappuis, V., Bornstein, M. M., Wittneben, J. G., Frei, M. \& Belser, U. C. (2013) Long-term stability of contour augmentation with early implant placement following single tooth extraction in the esthetic zone: a prospective, cross-sectional study in 41 patients with a 5- to 9-year follow-up. Journal of Periodontology 84: 1517-1527.

Cannizzaro, G., Torchio, C., Felice, P., Leone, M. \& Esposito, M. (2010) Immediate occlusal versus non-occlusal loading of single zirconia implants. A multicentre pragmatic randomised clinical trial. European Journal of Oral Implantology 3: 111-120.

Chiapasco, M. \& Zaniboni, M. (2009) Clinical outcomes of GBR procedures to correct periimplant dehiscences and fenestrations: a systematic review. Clinical Oral Implants Research 20 Suppl 4: 113-123.

Donath, K. \& Breuner, G. (1982) A method for the study of undecalcified bones and teeth with attached soft tissues. The Sage-Schliff (sawing and grinding) technique. Journal of Oral Pathology 11: 318-326.

Fischer, J., Schott, A. \& Martin, S. (2016) Surface micro-structuring of zirconia dental implants. Clinical Oral Implants Research 27: 162-166.

Gahlert, M., Roehling, S., Sprecher, C. M., Kniha, H., Milz, S. \& Bormann, K. (2012) In vivo performance of zirconia and titanium implants: a histomorphometric study in mini pig maxillae. Clinical Oral Implants Research 23: 281-286.

Gahlert, M., Rohling, S., Wieland, M., Sprecher, C. M., Kniha, H. \& Milz, S. (2009)

Osseointegration of zirconia and titanium dental implants: a histological and histomorphometrical study in the maxilla of pigs. Clinical Oral Implants Research 20: 12471253.

Hisbergues, M., Vendeville, S. \& Vendeville, P. (2009) Zirconia: Established facts and perspectives for a biomaterial in dental implantology. Journal of biomedical materials research. Part B, Applied biomaterials 88: 519-529.

Ichikawa, Y., Akagawa, Y., Nikai, H. \& Tsuru, H. (1992) Tissue compatibility and stability of a new zirconia ceramic in vivo. Journal of Prosthetic Dentistry 68: 322-326. 
Jensen, S. S. \& Terheyden, H. (2009) Bone augmentation procedures in localized defects in the alveolar ridge: clinical results with different bone grafts and bone-substitute materials. International Journal of Oral and Maxillofacial Implants 24 Suppl: 218-236.

Jung, R. E., Benic, G. I., Scherrer, D. \& Hammerle, C. H. (2015) Cone beam computed tomography evaluation of regenerated buccal bone 5 years after simultaneous implant placement and guided bone regeneration procedures--a randomized, controlled clinical trial. Clinical Oral Implants Research 26: 28-34.

Jung, R. E., Fenner, N., Hammerle, C. H. \& Zitzmann, N. U. (2013) Long-term outcome of implants placed with guided bone regeneration (GBR) using resorbable and non-resorbable membranes after 12-14 years. Clinical Oral Implants Research 24: 1065-1073.

Jung, R. E., Grohmann, P., Sailer, I., Steinhart, Y. N., Feher, A., Hammerle, C., Strub, J. R. \& Kohal, R. (2016) Evaluation of a one-piece ceramic implant used for single-tooth replacement and three-unit fixed partial dentures: a prospective cohort clinical trial. Clinical Oral Implants Research 27: 751-761.

Kilkenny, C., Browne, W. J., Cuthill, I. C., Emerson, M. \& Altman, D. G. (2010) Improving bioscience research reporting: The ARRIVE guidelines for reporting animal research. $J$ Pharmacol Pharmacother 1: 94-99.

Koch, F. P., Weng, D., Kramer, S., Biesterfeld, S., Jahn-Eimermacher, A. \& Wagner, W. (2010) Osseointegration of one-piece zirconia implants compared with a titanium implant of identical design: a histomorphometric study in the dog. Clinical Oral Implants Research 21: 350-356.

Kohal, R. J., Baechle, M., Han, J. S., Hueren, D., Huebner, U. \& Butz, F. (2009) In vitro reaction of human osteoblasts on alumina-toughened zirconia. Clinical Oral Implants Research 20: 1265-1271.

Kohal, R. J., Knauf, M., Larsson, B., Sahlin, H. \& Butz, F. (2012) One-piece zirconia oral implants: one-year results from a prospective cohort study. 1. Single tooth replacement. Journal of Clinical Periodontology 39: 590-597.

Kohal, R. J., Patzelt, S. B., Butz, F. \& Sahlin, H. (2013) One-piece zirconia oral implants: one-year results from a prospective case series. 2 . Three-unit fixed dental prosthesis (FDP) reconstruction. Journal of Clinical Periodontology 40: 553-562.

Kohal, R. J., Weng, D., Bachle, M. \& Strub, J. R. (2004) Loaded custom-made zirconia and titanium implants show similar osseointegration: an animal experiment. Journal of Periodontology 75: 1262-1268.

Manzano, G., Herrero, L. R. \& Montero, J. (2014) Comparison of clinical performance of zirconia implants and titanium implants in animal models: a systematic review. International Journal of Oral and Maxillofacial Implants 29: 311-320.

Mir-Mari, J., Benic, G. I., Valmaseda-Castellon, E., Hammerle, C. H. \& Jung, R. E. (2016a) Influence of wound closure on the volume stability of particulate and non-particulate GBR materials: an in vitro cone-beam computed tomographic examination. Part II. Clinical Oral Implants Research: [Epub ahead of print].

Mir-Mari, J., Wui, H., Jung, R. E., Hammerle, C. H. \& Benic, G. I. (2016b) Influence of blinded wound closure on the volume stability of different GBR materials: an in vitro cone-beam computed tomographic examination. Clinical Oral Implants Research 27: 258-265. 
Nart, J., Barallat, L., Jimenez, D., Mestres, J., Gomez, A., Carrasco, M. A., Violant, D. \& Ruiz-Magaz, V. (2016) Radiographic and histological evaluation of deproteinized bovine bone mineral vs. deproteinized bovine bone mineral with $10 \%$ collagen in ridge preservation. A randomized controlled clinical trial. Clinical Oral Implants Research: [Epub ahead of print].

Oliva, J., Oliva, X. \& Oliva, J. D. (2010) Five-year success rate of 831 consecutively placed Zirconia dental implants in humans: a comparison of three different rough surfaces. International Journal of Oral and Maxillofacial Implants 25: 336-344.

Payer, M., Arnetzl, V., Kirmeier, R., Koller, M., Arnetzl, G. \& Jakse, N. (2013) Immediate provisional restoration of single-piece zirconia implants: a prospective case series - results after 24 months of clinical function. Clinical Oral Implants Research 24: 569-575.

Payer, M., Heschl, A., Koller, M., Arnetzl, G., Lorenzoni, M. \& Jakse, N. (2015) All-ceramic restoration of zirconia two-piece implants--a randomized controlled clinical trial. Clinical Oral Implants Research 26: 371-376.

Piconi, C., Burger, W., Richter, H. G., Cittadini, A., Maccauro, G., Covacci, V., Bruzzese, N., Ricci, G. A. \& Marmo, E. (1998) Y-TZP ceramics for artificial joint replacements. Biomaterials 19: $1489-1494$.

Schenk, R. K., Olah, A. J. \& Hermann, W. (1984) Preparation of calcified tissues for light microscopy In: G. R. Dickson, ed. Methods of calcified tissue preparations. Amsterdam: Elsevier:: 1-56.

Schliephake, H., Hefti, T., Schlottig, F., Gedet, P. \& Staedt, H. (2010) Mechanical anchorage and peri-implant bone formation of surface-modified zirconia in minipigs. Journal of Clinical Periodontology 37: 818-828.

Schwarz, F., Herten, M., Ferrari, D., Wieland, M., Schmitz, L., Engelhardt, E. \& Becker, J. (2007) Guided bone regeneration at dehiscence-type defects using biphasic hydroxyapatite + beta tricalcium phosphate (Bone Ceramic) or a collagen-coated natural bone mineral (BioOss Collagen): an immunohistochemical study in dogs. International Journal of Oral and Maxillofacial Surgery 36: 1198-1206.

Stadlinger, B., Hennig, M., Eckelt, U., Kuhlisch, E. \& Mai, R. (2010) Comparison of zirconia and titanium implants after a short healing period. A pilot study in minipigs. International Journal of Oral and Maxillofacial Surgery 39: 585-592.

Strietzel, F. P., Khongkhunthian, P., Khattiya, R., Patchanee, P. \& Reichart, P. A. (2006) Healing pattern of bone defects covered by different membrane types--a histologic study in the porcine mandible. Journal of biomedical materials research. Part B, Applied biomaterials 78: $35-46$.

Thoma, D. S., Benic, G. I., Munoz, F., Kohal, R., Sanz Martin, I., Cantalapiedra, A. G., Hammerle, C. H. \& Jung, R. E. (2015) Histological analysis of loaded zirconia and titanium dental implants: an experimental study in the dog mandible. Journal of Clinical Periodontology 42: 967-975.

Wong, R. W. \& Rabie, A. B. (2010) Effect of bio-oss collagen and collagen matrix on bone formation. Open Biomed Eng J 4: 71-76.

Zellin, G., Gritli-Linde, A. \& Linde, A. (1995) Healing of mandibular defects with different biodegradable and non-biodegradable membranes: an experimental study in rats.

Biomaterials 16: 601-609. 
Zitzmann, N. U., Scharer, P. \& Marinello, C. P. (2001) Long-term results of implants treated with guided bone regeneration: a 5-year prospective study. International Journal of Oral and Maxillofacial Implants 16: 355-366.

Zumstein, T., Billstrom, C. \& Sennerby, L. (2012) A 4- to 5-year retrospective clinical and radiographic study of Neoss implants placed with or without GBR procedures. Clinical Implant Dentistry and Related Research 14: 480-490. 

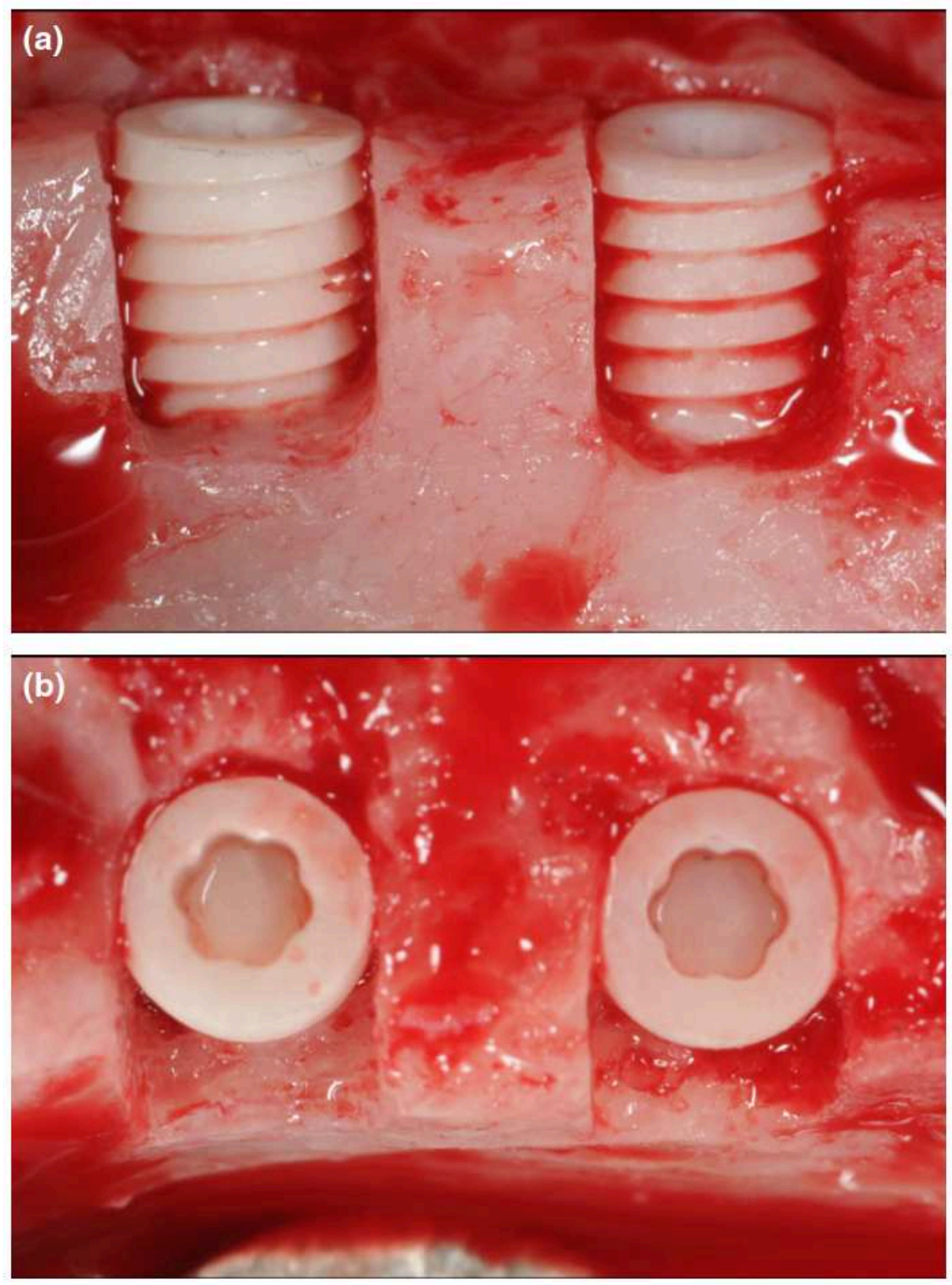

Figure 1 

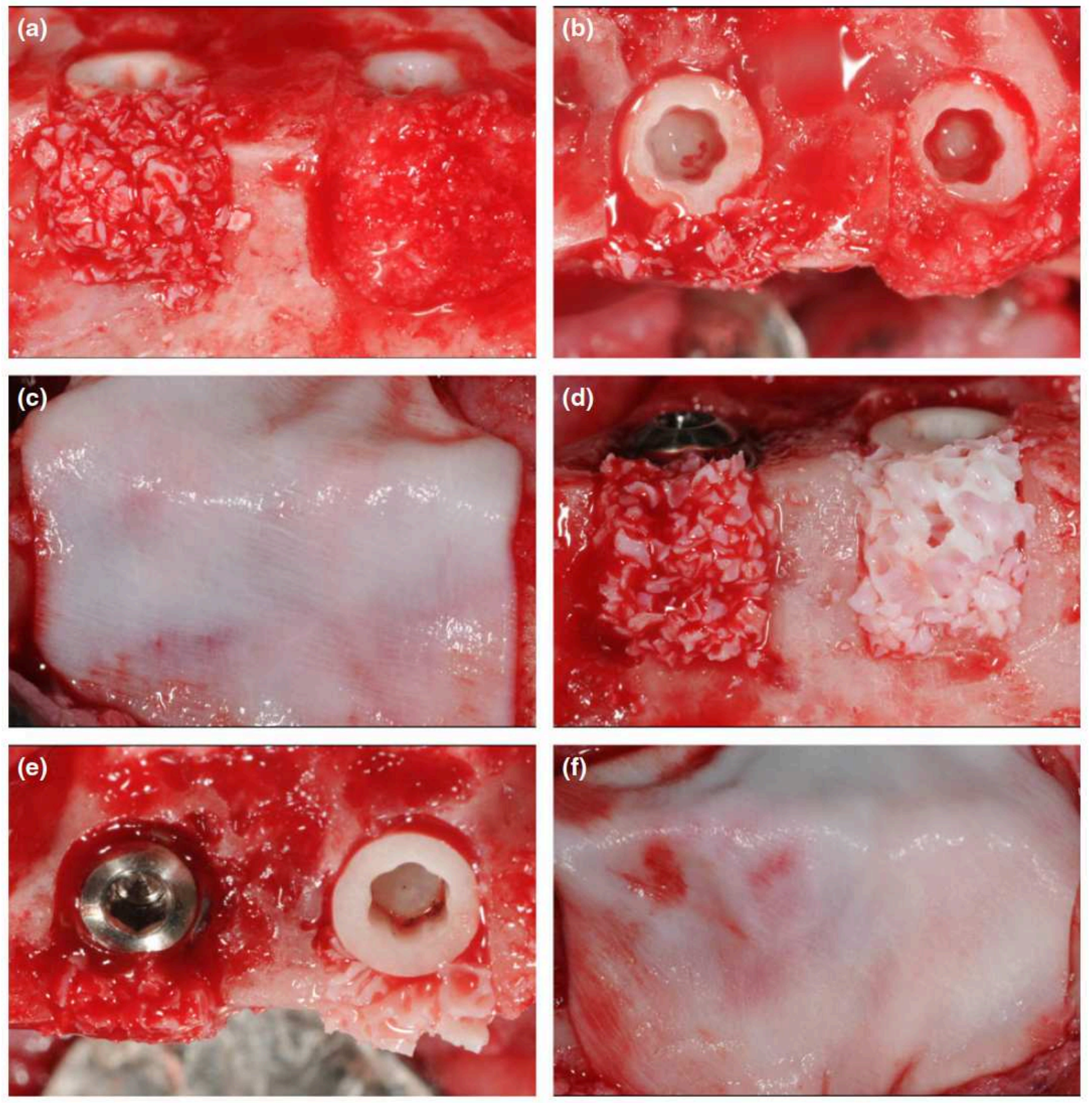

Figure 2 

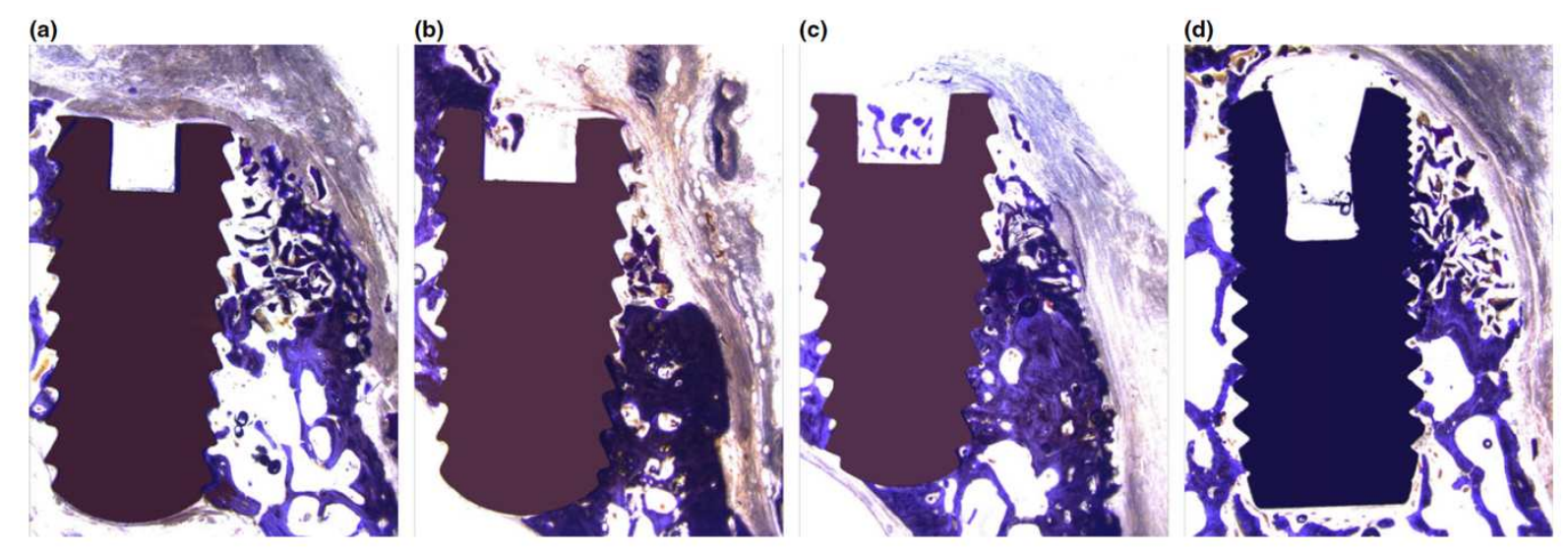

Figure 3

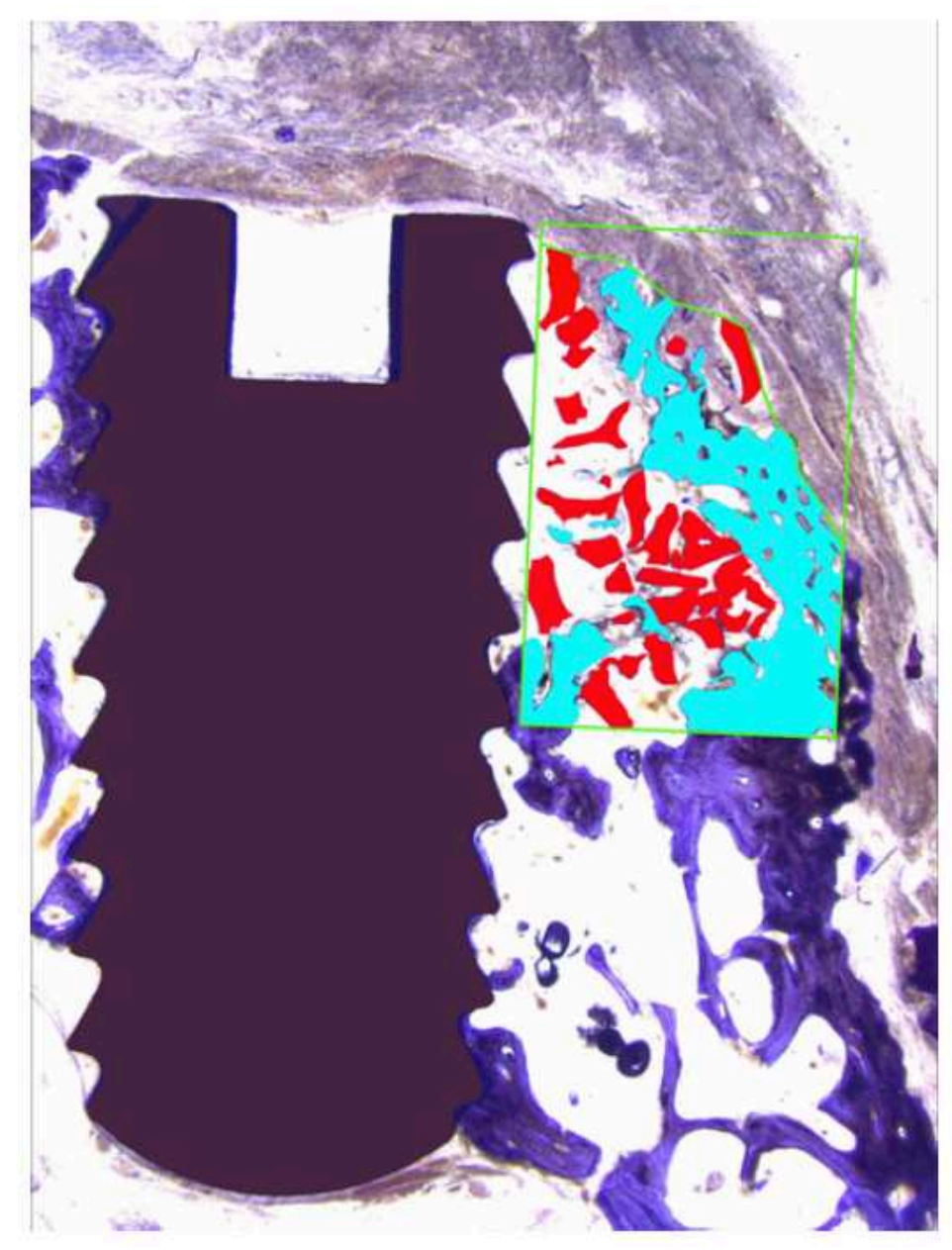

Figure 4 

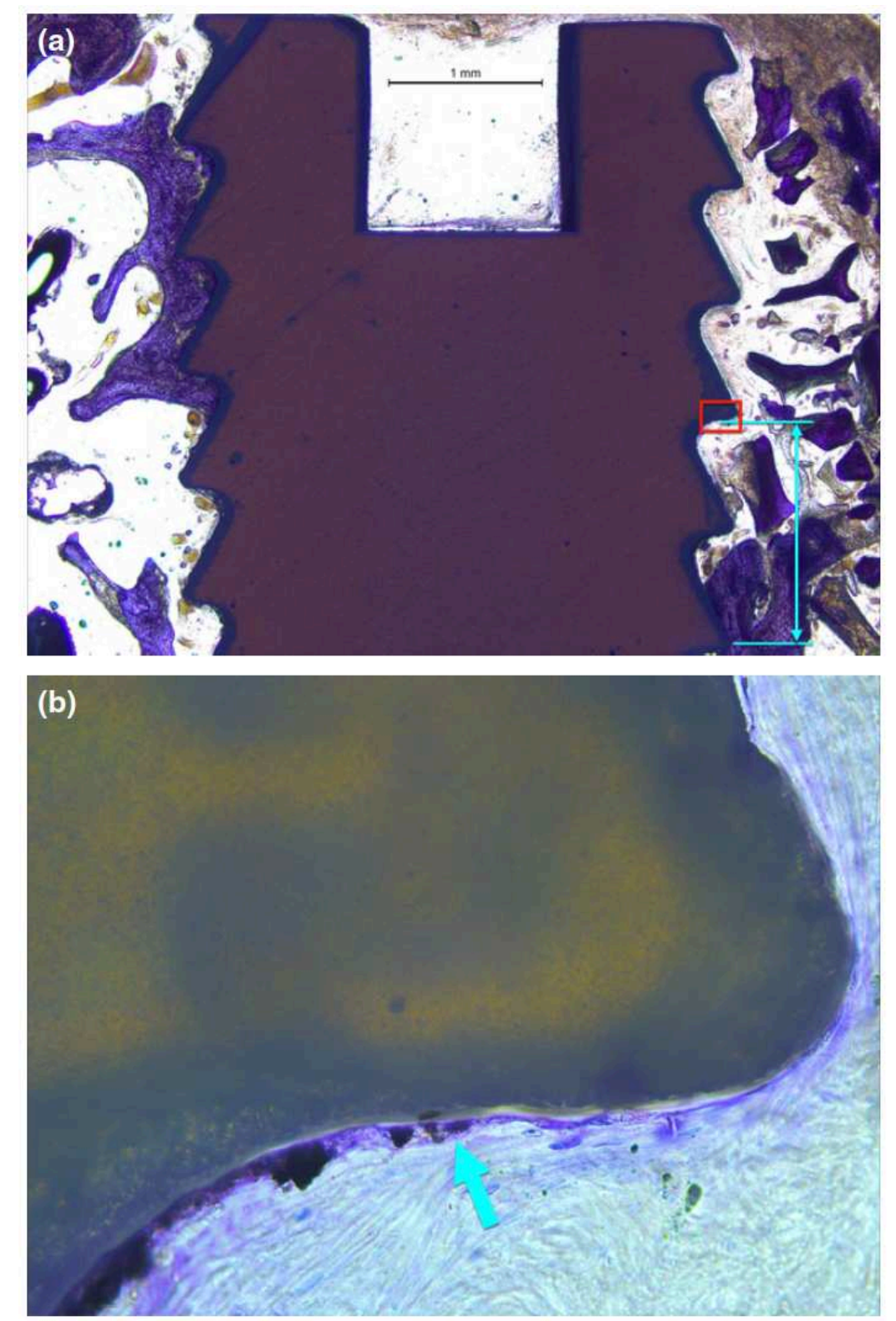

Figure 5 


\begin{tabular}{|c|c|c|c|c|c|}
\hline (a) & & $\begin{array}{l}\text { Treatment } \\
\text { modality }\end{array}$ & & & \\
\hline \multirow[t]{2}{*}{$\begin{array}{l}\text { Parame } \\
\text { ter }\end{array}$} & Unit & $\begin{array}{l}\text { 1. ZrO2 } \\
\text { implant + } \\
\text { DBBM } \\
\text { granules } \\
(\mathrm{n}=7)\end{array}$ & $\begin{array}{l}\text { 2. ZrO2 } \\
\text { implant + } \\
\text { DBBM- } \\
\text { collagen } \\
(n=5)\end{array}$ & $\begin{array}{l}\text { 3. ZrO2 } \\
\text { implant + } \\
\text { DBBM block } \\
(n=6)\end{array}$ & $\begin{array}{l}\text { 4. Ti implant } \\
+\quad \text { DBBM } \\
\text { granules } \\
(n=7)\end{array}$ \\
\hline & & $\begin{array}{l}\text { Mean } \pm \text { SD } \\
\text { (Q1, median, } \\
\text { Q3) }\end{array}$ & & & \\
\hline \multirow[t]{2}{*}{ AA } & $\mathrm{mm} 2$ & $8.6 \pm 4.0$ & $4.7 \pm 1.6$ & $5.1 \pm 1.9$ & $7.6 \pm 2.8$ \\
\hline & & $\begin{array}{l}(4.9,8.7 \\
12.3)\end{array}$ & $(3.2,4.4,6.4)$ & $(3.9,5.7,6.5)$ & $\begin{array}{l}(4.5,8.1 \\
10.2)\end{array}$ \\
\hline \multirow[t]{2}{*}{ NB } & $\mathrm{mm} 2$ & $2.0 \pm 1.7$ & $0.9 \pm 0.9$ & $2.1 \pm 0.9$ & $0.8 \pm 0.6$ \\
\hline & & $(0.6,1.4,4.3)$ & $(0.2,0.3,1.9)$ & $(1.3,2.2,2.9)$ & $(0.4,0.5,0.8)$ \\
\hline \multirow[t]{2}{*}{ BS } & $\mathrm{mm} 2$ & $2.3 \pm 1.6$ & $1.9 \pm 1.7$ & $1.0 \pm 0.6$ & $2.4 \pm 1.6$ \\
\hline & & $(1.2,1.8,4.4)$ & $(0.8,1.6,3.2)$ & $(0.6,0.8,1.5)$ & $(1.4,1.6,3.8)$ \\
\hline \multirow[t]{2}{*}{ NMT } & $\mathrm{mm} 2$ & $4.2 \pm 2.4$ & $1.9 \pm 1.7$ & $2.4 \pm 0.9$ & $4.4 \pm 1.4$ \\
\hline & & $(2.2,4.4,6.5)$ & $(0.9,1.2,3.2)$ & $(1.8,2.3,2.9)$ & $(3.0,4.7,5.7)$ \\
\hline \multirow{2}{*}{$\begin{array}{l}\text { DEF- } \\
\text { fBIC }\end{array}$} & $\mathrm{mm}$ & $2.1 \pm 1.7$ & $2.7 \pm 1.1$ & $2.9 \pm 1.2$ & $3.4 \pm 0.4$ \\
\hline & & $(0.3,1.5,3.4)$ & $(1.8,2.7,3.6)$ & $(2.0,2.7,4.0)$ & $(3.2,3.5,3.7)$ \\
\hline \multirow[t]{2}{*}{ BIC } & $\%$ & $70 \pm 19$ & $69 \pm 22$ & $77 \pm 30$ & $66 \pm 27$ \\
\hline & & $(52,74,79)$ & $(51,62,91)$ & $(42,91,100)$ & $(49,59,100)$ \\
\hline
\end{tabular}




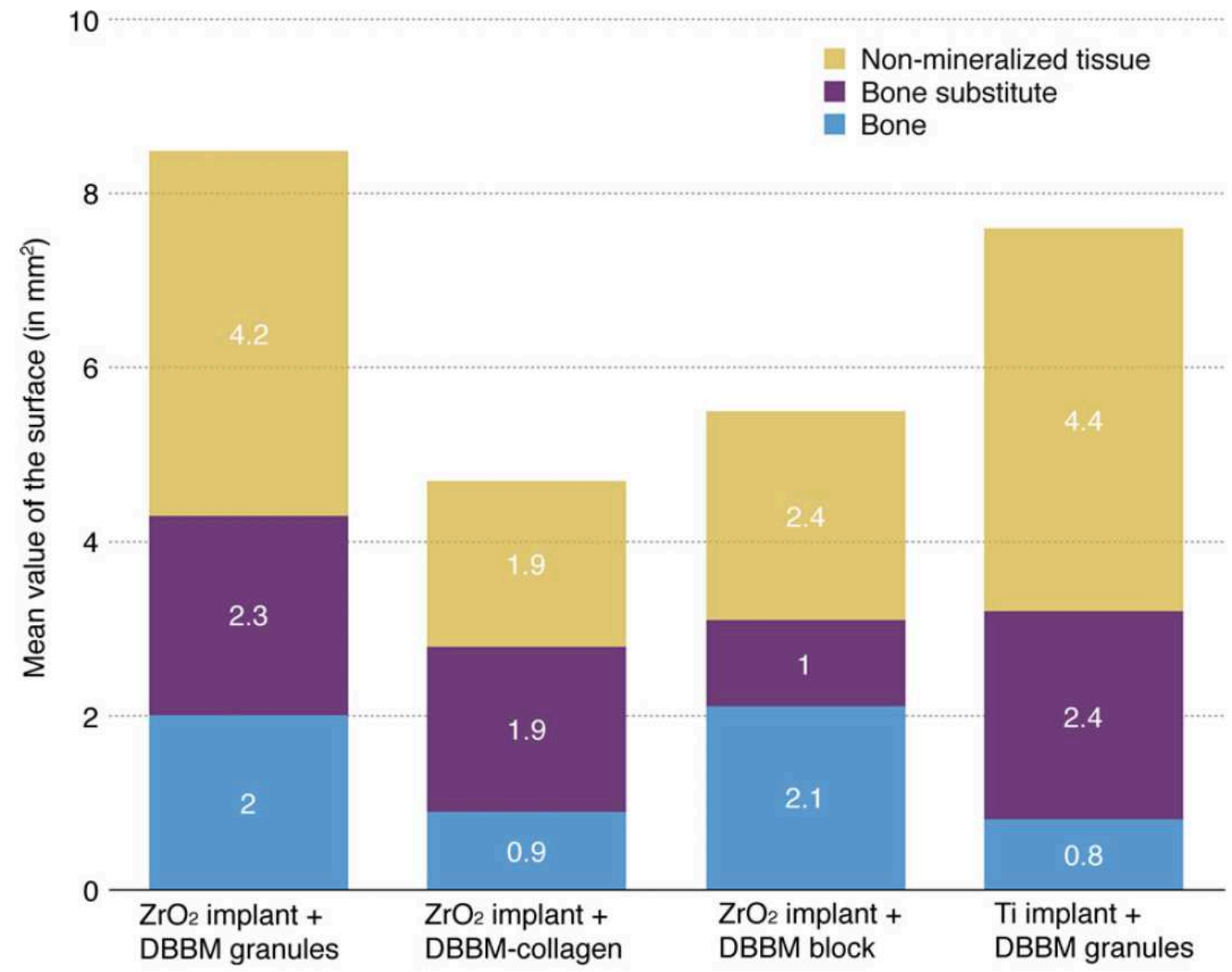

Figure 6 